\title{
Criptococose pulmonar e neurocriptococose em paciente imunocompetente: relato de caso
}

\author{
Pulmonary cryptocochosis and neurocyphococosis in immunocompetent patients: case \\ report
}

Criptococosis pulmonar y neurocriptococosis en paciente inmunocompetente: relato de caso

Lorena Santiago Monteiro Xavier ${ }^{1}$, Rodrigo Bona Maneschy ${ }^{1}$, Maristela Bacellar Cruz Nunes ${ }^{1}$, Leonardo Cruz Xavier ${ }^{1}$, Nina Pinto Monteiro Rocha ${ }^{1}$, Carla Daniele Nascimento Pontes ${ }^{1 *}$, Lucianna Serfaty de Holanda ${ }^{1}$, Bianca Luiza Silva de Aguiar ${ }^{1}$, Leonardo Santos da Silva ${ }^{1}$, Marilia Vieira Miranda ${ }^{1}$, Edvaldo Souza de Oliveira Junior ${ }^{1}$, Aline Monte Santos ${ }^{1}$, Luis Gustavo Silva Lima ${ }^{1}$, Yuri Jose Almeida da Silva', Beatriz Freitas do Prado".

\section{RESUMO}

Objetivo: Relatar o caso de um paciente com Criptococose Pulmonar em paciente imunocompetente. Relato de caso: Homem de 38 anos, com história de tosse seca há seis meses associada à febre vespertina diária e dor torácica ventilatório-dependente em hemitórax direito, cuja biópsia de material de lavado broncoalveolar sugeriu tratar-se de Criptococose. Apresentou, ainda, queixa de perda ponderal de $5 \mathrm{~kg}$ no período. Discussão: A Criptococose pulmonar, apesar de rara, deve fazer parte do diagnóstico diferencial entre os pacientes com história tosse seca associada à febre, mesmo em pacientes sem história epidemiológica sugestiva ou motivo de suspeição para imunodepressão.

Palavras-Chave: Criptococose, Imunocompetente, Neurocriptococose.

\begin{abstract}
Objective: To report the case of a patient with pulmonary cryptococcosis in an immunocompetent patient. Case report: A 38-year-old man with a history of dry cough for six months associated with daily evening fever and chest-dependent chest pain in the right hemithorax, whose biopsy of bronchoalveolar lavage material suggested that it was Cryptococcosis. She also presented a weight loss of $5 \mathrm{~kg}$ in the period. Discussion: Pulmonary Cryptococcosis, although rare, should be part of the differential diagnosis among patients with a history of dry cough associated with fever, even in patients with no suggestive epidemiological history or suspected immunodepression.
\end{abstract}

Key words: Cryptococcosis, Immunocompetent, Neurocryptococcosis.

\section{RESUMEN}

Objetivo: Informar el caso de un paciente con Cripitococosis Pulmonar en paciente inmunocompetente. Relato de caso: Hombre de 38 años, con historia de tos seca hace seis meses asociada a la fiebre vespertina diaria y dolor torácica ventilatorio-dependiente en hemitórax derecho, cuya biopsia de material de lavado broncoalveolar sugirió tratarse de Criptococosis. Se presentó, además, una queja de pérdida ponderal de 5 $\mathrm{kg}$ en el período. Discusión: La Criptococosis pulmonar, a pesar de rara, debe formar parte del diagnóstico diferencial entre los pacientes con historia tos seca asociada a la fiebre, incluso en pacientes sin historia epidemiológica sugestiva o motivo de sospecha para inmunodepresión.

Palabras clave: Criptococosis, Inmunocompetente, Neurocriptococosis.

${ }^{1}$ Fundação Santa Casa de Misericórdia do Pará. *E-mail: pontes4@live.com

SUBMETIDO EM: 10/2018

ACEITO EM: 11/2018

PUBLICADO EM: $3 / 2019$ 


\section{INTRODUÇÃO}

A criptococose é uma infecção causada por um basidiomiceto encapsulado do gênero Cryptococcus, o qual é um fungo oportunista que tem ganhado notável relevância no sistema de saúde (CHAYAKULKEEREE M e PERFECT JR, 2006). Com a epidemia de HIV houve um aumento marcante no número de casos de Criptococose registrados em todo o mundo, em especial pelas espécies C. neoformans e C. gattii (KIDD SE et al 2004).

A criptococose é a micose sistêmica que gerou maior número de internações em todo o país dos anos de 2000 a 2007 devido sua característica oportunística. Nas regiões Norte e Nordeste do Brasil predominam casos de criptococose em indivíduos sem evidência de imunodepressão, independente do gênero causados pela variedade C. gattii (BRASIL 2012). A infecção ocorre após inalação de leveduras ou basidiósporos e é facilitada pelo diâmetro $(1,2-1,8 \mu \mathrm{m})$ desses últimos, o qual é pequeno o suficiente para que haja acumulo no alvéolo e desenvolvimento até a forma de levedura (COHEN J et al, 1982; CONCEIÇÃO MS et al, 2013).

O hospedeiro interage com o fungo e gera o chamado complexo linfonodal pulmonar primário. Inicialmente a infecção é assintomática e autolimitada, especialmente se o hospedeiro estiver com o sistema imune intacto para combater o fungo. Porém, as leveduras podem ficar latentes dentro do complexo linfonodal até sua morte ou até uma imunossupressão que permita sua reativação e consequente infecção (ZIMMER BL et al. 1984; PANTOJA MJL, SILVEIRA DMR E SILVA LD, 2009).

O fungo encontra no SNC uma reunião de nutrientes que o atraem, como tiamina, glutamina, carboidratos, entre outros que encontra no líquor, associado a baixa resposta inflamatória e baixa atuação do complemento nesse sistema, por conta disso, nas fases de disseminação as principais manifestações são pulmonares e neurológicas (LITTMAN ML, 1959; PANTOJA MGL et al 2009; CONCEIÇÃO MS et al, 2013). O tratamento da cripitococose é baseado na erradicação fúngica com uso de anti-fúngicos endovenosos e/ou orais e os principais agentes disponíveis são Anfotericina B, Anfotericina Lipossomal, Itraconazol e Fluocitosina (CONCENSO DE CRIPTOCOCOSE 2008).

\section{DESCRIÇÃO DO CASO}

Paciente A.M.S, do sexo masculino, 38 anos, admitido na Fundação Santa Casa de Misericórdia do Pará (FSCMPA), procedente de Anajás/PA, referiu que em agosto de 2016 iniciou quadro de tosse seca associado a febre vespertina, não aferida, diária, além de dor torácica ventilatório-dependente em hemitórax direito, além de perda ponderal de $5 \mathrm{~kg}$ entre os meses de agosto de 2016 e fevereiro de 2017.

Ainda no seu município de origem, após realização de radiografia, recebeu o diagnóstico de pneumonia e iniciou tratamento com antibiótico, via oral, sem melhora clínica, mesmo que parcial. Em seguida, foi encaminhado para o ambulatório de pneumologia da FSCMPA, em Belém, onde os resultados dos exames descritos seguem em ordem cronológica:

Na Figura 1, foi realizado uma TC de tórax no dia 20/10/2016 e apresentou massa com atenuação de partes moles, heterogênea, parcialmente definidos, contornos irregulares, localizado em seguimento medial do lobo médio, medindo $6 \times 6,7 \times 4,8 \mathrm{~cm}$, com pequenos nódulos perilinfáticos satélites e árvore em brotamento no parênquima adjacente. Estrias fibroelásticas em ápices pulmonares.

BRONCOSCOPIA (05/12/2016): Lesão obstrutiva segmentar medial.

CITOLOGIA DO LAVADO BRONCOALVEOLAR: Sedimentos com alterações degenerativas e leucócitos sugestivos de presença de processo inflamatório. Pesquisa de fungos: presença de leveduras encapsuladas sugestivas de Cryptococcus Sp.

TESTE DE HIV 1 e 2 (10/12/2016): Negativo

HISTOPATOLÓGICO (16/12/2016): Quadro morfológico compatível com Criptococose. 
Figura 1 - TC de tórax (20/10/16).

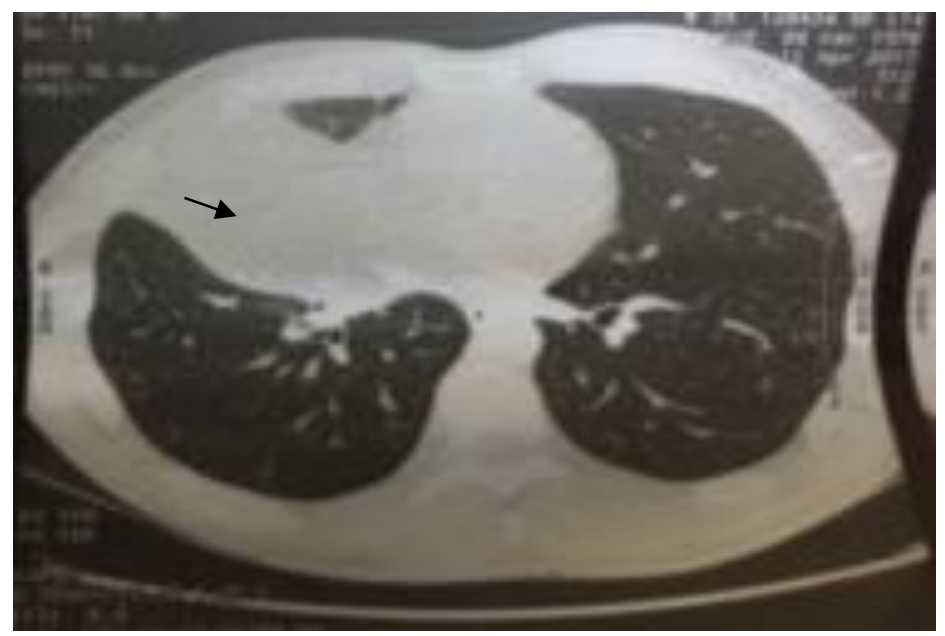

Fonte: Dados Primários, 2016

Diante de tais achados, a pneumologista responsável pelo paciente iniciou tratamento com Fluconazol 300 $\mathrm{mg} / \mathrm{dia}$. e emitiu guia de Internação de Hospitalar para início de tratamento com Anfotericina B em virtude da extensão da lesão e riqueza de sinais e sintomas apresentados pelo paciente.

No dia 18/02/2017 o paciente foi internado, apresentando-se, ao exame físico, estado geral bom, psiquismo normal, palidez cutâneo-mucosa +/++++, dispneico, hidratado, acianótico, afebril, anictérico. $\mathrm{Na}$ avaliação do aparelho respiratório, FR de 24 irpm; à percussão, maciçez (porção inferior e média em HTD); à ausculta, murmúrio vesicular diminuído em HTD, preservado em HTE, sem ruídos adventícios. Exame cardiovalscular e abdômen sem alterações, com lifonodomegalia em região cervica direita.

No dia seguinte à internação, iniciou tratamento com a Anfoterina $B, 50 \mathrm{mg} / \mathrm{dia}$, ao longo de 60 dias, com dose cumulativa de $2 \mathrm{~g}$ ao final. Além disso, realizou nova sorologia para HIV, foi feita nova requisição de TC de Tórax e Crânio, além da investigação de possíveis causas farmacológicas para imunossupressão e exames de laboratorias gerais, como hemograma, dosagens de escórias nitrogenadas, transaminases, perfil glicêmico, investigação de dislipidemias e sorologias para hepatites virais, os resultados dos exames laboratoriais de rotina estavam dentro do padrão de normalidade.

Durante os 2 meses de internação, em uso de Anfotericina $B$, o paciente evoluiu em vários momentos com hipocalemia, precisando fazer reposição de $\mathrm{KCl} 10 \%$. Foram solicitados, ainda, os exames complementares, descritos a seguir, com seus respectivos resultados:

TESTE DE HIV 1 e 2 (26/02/2017): Negativo

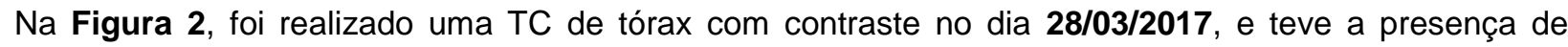
formação arredondada, de paredes espessas, com densidade heterogênea, de atenuação líquida espessa de partes moles, com septações internas grosseiras com realce parietal homogêneo após injeção de contraste, localizada no lobo médio, medindo cerca de $8,7 \times, 5 \times 1,6 \mathrm{~cm}$. Nota - se, ainda, espessamento parasseptal adjacente. Ausência de derrame pleural e não se identificam linfonodomegalias mediastinais.

PESQUISA DE LCR: negativa.

EXAME ANATOMO-PATOLÓGICO (20/04/2017): Lobo pulmonar medindo $20 \times 10 \times 6 \mathrm{~cm}$, com espessamento pleural ao nível da periferia romboide e outras áreas cinza escuras. Observamos ao nível da ressecção exteriorização de material mucoide, onde aos cortes evidenciamos nodulação pardo clara mucoide $\mathrm{cm}$ parcial anel brancacento medindo $8 \mathrm{~cm}$ de diâmetro. Conclusão após avaliação microscópica: Criptococose pulmonar (Toruloma). 
Figura 2 - TC de tórax com contraste (28/03/2017).

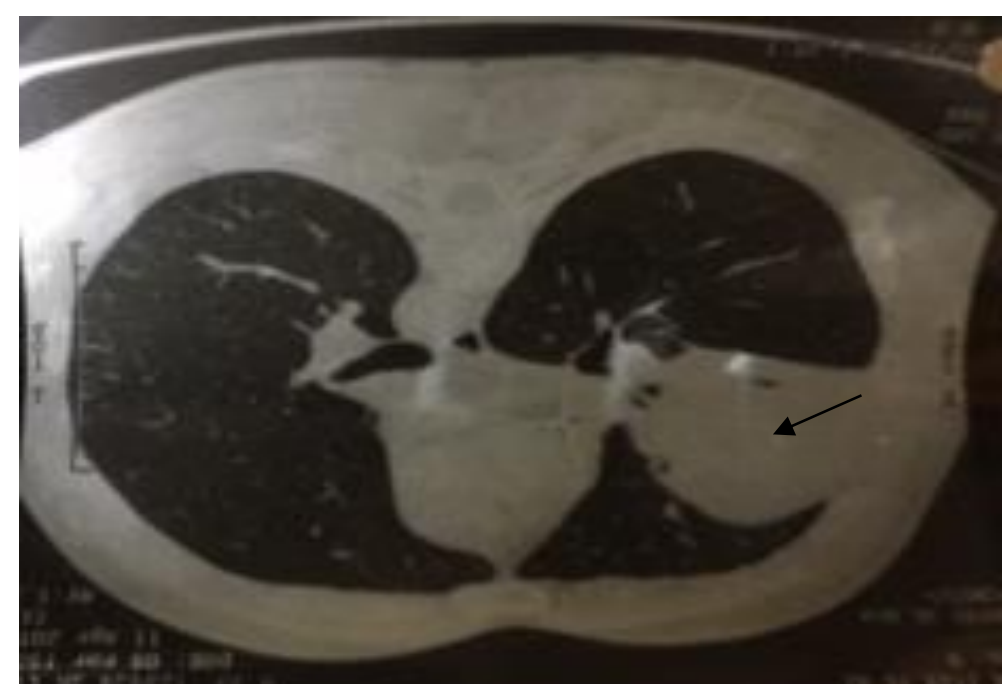

Fonte: Dados Primários, 2017.

Ao final do tratamento, não houve melhora clínica significativa, observando-se inclusive piora radiológica. Paciente foi, então, foi reavaliado pela pneumologista que interpretou como inefetivo o tratamento realizado, indicando, a partir daí, a realização de bilobectomia a ser realizada pelo cirurgião Torácico.

Paciente foi submetido à Bilobectomia médio e inferior direita, no dia 19/04/2017, tendo realizado a cirurgia sem relato de intercorrências, no pós cirúrgico, necessitou receber no total quatro unidades de concentrados de hemácias, ficando internado durante nove dias em UTI, onde evoluiu com melhora, retornando a enfermaria, onde permaneceu por mais 04 dias, tendo posteriormente recebido a alta.

Paciente segue acompanhado nos ambulatórios de pneumologia e neurocirurgia, fazendo uso de Fluconazol $400 \mathrm{mg} / \mathrm{dia}$. Com relação a neurocriptococose, além do uso do antifúngico, a programação de realização de novo exame de imagem dentro de três meses, para reavaliação de conduta, até o momento não invasiva.

\section{DISCUSSÃO}

A infecção por C. neoformans e C. gattii acomete prioritariamente o sistema nervoso central e órgãos respiratórios mas pode acometer demais órgãos e sistemas como pele, próstata, trato urinário, ossos e globo ocular (THOMPSON JC et al, 2006).

Historicamente a cripitococose estava associada a imunodeficiências como uso crônico de corticoide, diabetes mellitus e deficiências de imunoglobulinas, mas, ganhou forças após a epidemia de HIV. Porém, nos últimos anos houve um aumento do número de casos, mesmo em pacientes imunocompetentes (CONSENSO EM CRIPTOCOCOSE: 2008).

Pacientes imunocomprometidos com criptococose são os que mais apresentam mais frequentemente manifestações no SNC do que nos pulmões (HEMPEL et al 1984; THOMPSON et al 2006). Em contrapartida, nos pacientes imunocompetentes o envolvimento pulmonar é menor frequente (CHAYAKULKEEREE $M e$ PERFECT JR, 2006).

No estudo do paciente em questão, não se detectou no acompanhamento ambulatorial e nem durante a internação evidências de imunodepressão. Ainda assim, ratificando a raridade do evento descrito (CHAYAKULKEEREE M e PERFECT JR, 2006), observou-se acometimento do SNC radiologicamente, embora, até o momento não haja manifestações clínicas de comprometimento nervoso. 
Os preceitos do tratamento preconizados na literatura para os casos de Criptococose pulmonar isolada, com tratamento inicial com Fluconazol, via oral, $200-400 \mathrm{mg} /$ dia, foram seguidos. Porém, diante da extensão da lesão no parênquima pulmonar, dos sintomas de desconforto respiratório, e da dificuldade de acompanhamento do paciente ambulatorialmente, visto que, trata-se de um morador de ambiente rural, muito distante da capital, o pedido de internação e início do tratamento com a Anfotericina B foram antecipados (KIDD et al., 2004).

Atualmente, a ressecção cirúrgica não é recomendada de forma rotineira, exceto nas seguintes condições: lesões pseudotumorais e não responsivas ao tratamento clínico e em casos com derrame pleural intenso e exsudativo em que há a necessidade de drenagem torácica e até decorticação. Lobectomia parcial ou completa são realizadas, em muitos casos, em virtude da necessidade de diagnóstico diferencial com neoplasias. Nestes pacientes, a ressecção é considerada como medida terapêutica suficiente para a resolução da doença (CONSENSO EM CRIPTOCOCOSE, 2008).

Dessa forma, a lobectomia no caso em questão veio a ser a última linha de tratamento a ser realizada, como instrumento para resolução da Criptococose pulmonar, ficando o paciente em acompanhamento ambulatorial para neurocriptococose.

A Criptococose pulmonar, apesar de rara, deve fazer parte do diagnóstico diferencial entre os pacientes com história tosse seca associada à febre, mesmo em indivíduos sem história epidemiológica sugestiva ou com motivo de suspeição para imunodepressão. Nesse caso específico, o diagnóstico precoce, através de uma maior suspeição poderiam ter modificado a história natural da doença.

\section{REFERÊNCIAS}

1. CHAYAKULKEEREE M, PERFECT JR. Cryptococcosis. Infect Dis Clin North Am. 2006;20(3):507-44, v-vi.

2. KIDD SE, HAGEN F, TSCHARKE RL, et al. A rare genotype of Cryptococcus gattii caused the cryptococcosis outbreak on Vancouver Island (British Columbia, Canada). Proc Natl Acad Sci USA. 2004;101(49):17258-63.

3. CONSENSO EM CRIPTOCOCOSE: 2008. Rev. Soc. Bras. Med. Trop. [Internet]. 2008 Oct [cited 2017 Sep 03]; 41 (5): 524-544.

4. COHEN J, PERFECT JR, DURACK DT. Cryptococcosis and the basidiospore. Lancet. 1982;1(8284):1301.

5. ZIMMER BL, HEMPEL HO, GOODMAN NL. Pathogenicity of the basidiospores of Filobasidiella neoformans. Mycopathologia. 1984;85(3):149-53.

6. COHEN J. The pathogenesis of cryptococcosis. J Infect. 1982;5(2):109-16.

7. LITTMAN ML, SCHNEIERSON SS. Cryptococcus neoformans in pigeon excreta in New York City. Am J Hyg. 1959;69(1):49-59.

8. THOMPSON JC, KISHIMA M, GOMES, et al. Proteinose alveolar pulmonar: série de quatro casos. Jornal Brasileiro de Pneumologia, 32(3), 261-266 - 2006.

9. CONCEIÇÃO MS, SILVA ILF, SGANZERLA BMM. Proteinose alveolar pulmonar associada a comprometimento do sistema nervoso central por criptococo - relato de caso. Revista Paraense de Medicina - V.27 (3) julhosetembro 2013.

10. PANTOJA MGL, SILVEIRA DMR, SILVA LD. Criptococose disseminada em paciente imunocompetente: relato de caso. Rev. para. med = Rev. Para. Med. (Impr.);23(3), jul.-set. 2009.

11. BRASIL. Vigilância e Epidemiologia da Criptococose. Ministério da Saúde - Brasilia - DF - Abril de 2012. 